\title{
Pseudotyped Vesicular Stomatitis Virus for Analysis of Virus Entry Mediated by SARS Coronavirus Spike Proteins
}

\author{
Shuetsu Fukushi, Rie Watanabe, and Fumihiro Taguchi
}

\begin{abstract}
Severe acute respiratory syndrome (SARS) coronavirus $(\mathrm{CoV})$ contains a spike (S) protein that binds to a receptor molecule (angiotensin-converting enzyme 2; ACE2), induces membrane fusion, and serves as a neutralizing epitope. To study the functions of the $S$ protein, we describe here the generation of SARS-CoV S protein-bearing vesicular stomatitis virus (VSV) pseudotype using a VSV $\Delta$ G*/GFP system in which the G gene is replaced by the green fluorescent protein (GFP) gene (VSV-SARS-CoV-St19/GFP). Partial deletion of the cytoplasmic domain of SARS-CoV S protein (SARS-CoV-St19) allowed efficient incorporation into the VSV particle that enabled the generation of a high titer of pseudotype virus. Neutralization assay with anti-SARS-CoV antibody revealed that VSV-SARS-St19/GFP pseudotype infection is mediated by SARS-CoV S protein. The $\mathrm{VSV} \Delta \mathrm{G}^{*} / \mathrm{SEAP}$ system, which secretes alkaline phosphatase instead of GFP, was also generated as a VSV pseudotype having SARS-CoV S protein (VSV-SARS-CoV-St19/SEAP). This system enabled high-throughput analysis of SARS-CoV S protein-mediated cell entry by measuring alkaline phosphatase activity. Thus, VSV pseudotyped with SARS$\mathrm{CoV} \mathrm{S}$ protein is useful for developing a rapid detection system for neutralizing antibody specific for SARS-CoV infection as well as studying the S-mediated cell entry of SARS-CoV.
\end{abstract}

Key words: SARS-CoV; coronavirus; pseudotype; vesicular stomatitis virus; spike protein; attachment; fusion; cell entry.

From: Methods in Molecular Biology, vol. 454: SARS- and Other Coronaviruses, Edited by: D. Cavanagh, DOI: 10.1007/978-1-59745-181-9_23, C Humana Press, New York, NY 


\section{Introduction}

Entry of SARS coronavirus (SARS-CoV) into target cells is mediated by binding of the viral spike (S) protein to the receptor molecules, angiotensinconverting-enzyme 2 (ACE2) (1). Studies on SARS using infectious SARS-CoV require care because of the highly pathogenic nature of this virus, so an alternative methodology is needed. Recently, pseudotyped retrovirus particles bearing SARS-CoV S protein have been generated by several laboratories (2-4). These pseudotyped viruses have been shown to have a cell tropism identical to authentic SARS-CoV and their infectivity is dependent on ACE2, indicating that the infection is mediated solely by SARS-CoV S protein. Pseudotyped viruses have proved to be a safe viral entry model because of an inability to produce infectious progeny virus. A quantitative assay of pseudovirus infection could facilitate the research on SARS-CoV entry, cell tropism, and neutralization antibody.

Another pseudotyping system with a vesicular stomatitis virus (VSV) particle was previously reported to produce pseudotypes of envelope glycoprotein of several RNA viruses (i.e., measles virus, hantavirus, Ebola virus, and hepatitis $\mathrm{C}$ virus) (5-8). This system (VSV $\Delta \mathrm{G}^{*} / \mathrm{GFP}$ system) may be useful for research on envelope glycoprotein owing to its ability to grow high titers in a variety of cell lines. The pseudotype virus titer obtained from the VSV $\Delta$ G*/GFP system $\left(>10^{5}\right.$ infectious units (IU)/ml) is generally higher than that of the pseudotyped retrovirus system (6). Furthermore, infection of pseudotyped VSV in target cells can be detected as GFP-positive cells within $16 \mathrm{~h}$ postinfection (hpi) because of the powerful GFP-expression in the VSV $\Delta \mathrm{G}^{*} / \mathrm{GFP}$ system (6). In contrast, the time required for the pseudotyped retrovirus system to detect infection is $48 \mathrm{hpi}$ $(\mathbf{9}, \mathbf{1 0})$, which is similar to that for the SARS-CoV to replicate to the level of producing plaques or cytopathic effects on infected cells. Thus, pseudotyping of SARS-CoV S protein using the VSV $\Delta \mathrm{G}^{*} / \mathrm{GFP}$ system may have greater advantages than retrovirus pseudotypes for studying the function of SARS-CoV $S$ protein as well as for developing a rapid system for detection of neutralizing antibody specific for SARS-CoV.

Here we describe protocols for introducing SARS-CoV-S protein into VSV particles using the VSV $\Delta$ G*/GFP system. The infection of VSV pseudotype bearing SARS-CoV S protein (VSV-SARS-St19/GFP) was easily detected in target cells as an expression of the GFP protein. The following methods were originally designed to produce VSV-SARS-St19/GFP and to measure the infection efficiency. In addition to a significant advantage of the VSV-SARS-St19/GFP for safe and rapid analyses of infection, the VSV $\Delta \mathrm{G}^{*} / \mathrm{SEAP}$ system, in which the $\mathrm{G}$ gene is replaced with the secreted alkaline phosphatase (SEAP) gene, may be superior to high-throughput quantitative analysis of S-mediated cell entry. The protocol for analyzing pseudotypes using the VSV $\Delta$ G*/SEAP system is also described briefly. 


\section{Materials}

1. Cell lines: The human embryonic kidney 293T (ATCC CRL-11268) is used to produce VSV pseudotypes bearing SARS-CoV-S proteins. The Vero E6 (ATCC Vero clone CRL 1586) is used for target cells of VSV pseudotype infection.

2. Dulbecco's Modified Eagle's Medium with high glucose concentration supplemented with 5\% fetal calf serum (DMEM-5\%FCS) is used for growing the 293T and Vero E6 cells.

3. Phosphate-buffered saline (PBS): $0.14 \mathrm{M} \mathrm{NaCl}, 2 \mathrm{mM} \mathrm{KCl}, 3 \mathrm{mM} \mathrm{Na} 2 \mathrm{HPO} 4$, $1.5 \mathrm{mM} \mathrm{KH}_{2} \mathrm{PO} 4, \mathrm{pH} 7.2$. Autoclave to sterilize it.

4. Polyfect transfection reagent (QIAGEN, Hilden, Germany)

5. Mammalian expression plasmid encoding SARS-CoV-S protein with 19 amino acid truncation in the $\mathrm{C}$ terminus (see Note $\mathbf{1}$ ).

6. The VSV $\Delta$ G*/GFP-G or VSV $\Delta$ G*/SEAP-G (see Note 2).

7. $0.22-\mu \mathrm{m}$ pore size sterile filter.

8. Digital fluorescence microscope for detecting GFP expression.

9. Reporter Assay Kit-SEAP (Toyobo, Osaka, Japan) and luminescence microplate reader for SEAP activity analyses.

\section{Methods}

A schematic description of the production of VSV pseudotype is shown in Fig. 1.

\subsection{Expression of SARS-CoV S Protein and Production of VSV Pseudotype}

1. Plate 293T cells onto a type I collagen-coated T-75 flask in DMEM-5\%FCS at a 20-30\% confluent (generally $2.5 \times 10^{6}$ cells per T-75 flask).

2. After $3 \mathrm{~h}$, transfect the $293 \mathrm{~T}$ cells with $18.5 \mu \mathrm{g}$ of expression plasmid encoding SARS-CoV-S protein (pKS/SARS-St19) using Polyfect transfection reagent according to the procedure recommended by the manufacturer. Incubate the cells in a $\mathrm{CO}_{2}$ incubator for $48 \mathrm{~h}$ in DMEM-5\% FCS. During this incubation, 293T cells grow to reach confluence.

3. Remove the supernatant from the cells. Wash the cells with PBS and add $10 \mathrm{ml}$ of fresh DMEM-5\%FBS. Inoculate the $1 \times 10^{6}$ IU of VSV $\Delta \mathrm{G}^{*} / \mathrm{GFP}-\mathrm{G}$ or VSV $\Delta \mathrm{G}^{*} / \mathrm{SEAP}-\mathrm{G}$ to produce pseudotypes to express GFP or SEAP, respectively. Incubate the cells in $\mathrm{CO}_{2}$ incubator for absorption.

4. After $1 \mathrm{~h}$, wash the cells with PBS three times. Then add the $15 \mathrm{ml}$ of fresh DMEM-5\%FCS.

5. After $24 \mathrm{~h}$, collect the culture supernatants that contain VSV pseudotype-bearing SARS-CoV S protein (VSV-SARS-St19/GFP or VSV-SARS-St19/SEAP).

6. Centrifuge the supernatants at $3000 \times \mathrm{g}$ for $5 \mathrm{~min}$ to remove cell debris, and then filter through a $0.22-\mu \mathrm{m}$ pore size filter. Store at $-80^{\circ} \mathrm{C}$. 
(5)

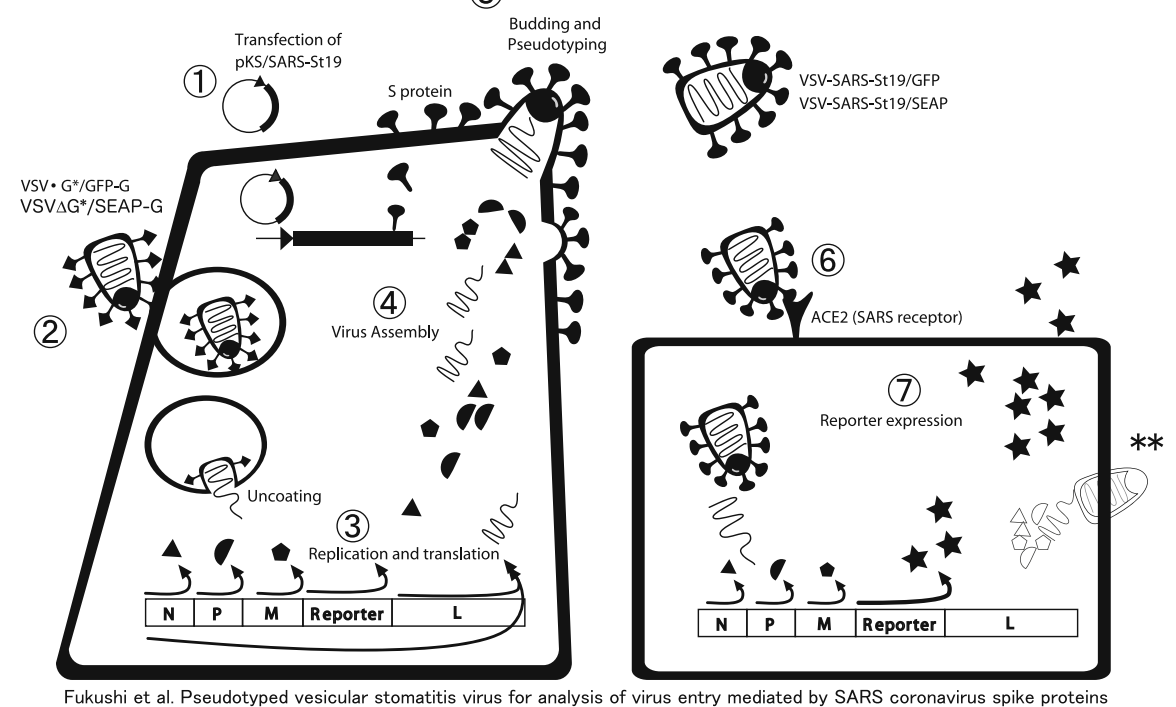

Fig. 1 Schematic illustration of the production and infection of VSV pseudotype bearing SARS-CoV S protein: (1) Transfection of pKS/SARS-St19. It provides the S protein of SARS-CoV and cells express the $S$ protein on the cell surface. (2) Infection of VSV $\Delta$ G*/GFP-G or VSV $\Delta \mathrm{G}^{*} / \mathrm{SEAP}-\mathrm{G}$. These viruses possess the genome containing the reporter gene instead of the VSV-G gene. (3) Virus replication and translation. All viral components except the $G$ protein will be supplied by these viruses. (4) Virus assembly, budding, and pseudotyping. Translated viral proteins are assembled and viral particles bud from the plasma membrane. Since the $S$ protein, which was provided by the expression plasmid, is expressed on the cell surface, a virus can incorporate it into the virus particle. (5) VSV-SARS-St19/GFP and VSV-SARS-St19/SEAP. Pseudotyped VSV possessing SARS-S protein is released into culture supernatant. (6) Infection of the pseudotyped VSV. These viral particles can infect cells expressing the receptor for SARS-CoV, ACE2. (7) Estimation of the infectivity of the viruses. The infection of the pseudotyped viruses is estimated by the expression level of the reporter protein. **Progeny viruses are produced by the infection of VSV-SARS-St19/GFP or VSVSARS-St19/SEAP. However, these viruses do not have infectivity because they have no glycoprotein (virus with the thin lines).

\subsection{Determining Infectivity of VSV-SARS-St19/GFP}

The infectivity of VSV-SARS-St19/GFP, harboring the VSV $\Delta$ G*/GFP genome, can be determined as the number of GFP-positive cells.

1. Mix serially diluted VSV-SARS-St19/GFP with DMEM-5\%FCS and inoculate the mixture into Vero E6 cells seeded in 96-well culture plates. 


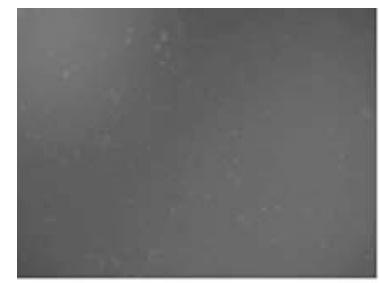

mock

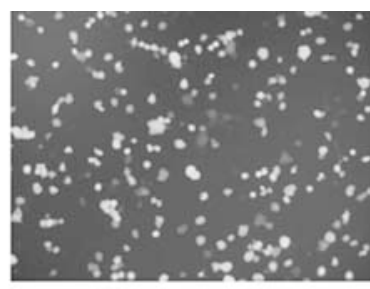

VSV-SARS-St19/GFP

Fig. 2. Detection of VSV-SARS-St19/GFP infection. The VSV-SARS-St19/GFP was inoculated to Vero E6 cells. GFP expression was examined under a fluorescent microscope.

2. Incubate the cells in a $\mathrm{CO}_{2}$ incubator for $7 \mathrm{~h}$. Longer incubation (overnight to $24 \mathrm{~h}$ ) may be beneficial for clearer fluorescent intensities on GFP-positive cells.

3. Detect GFP-positive cells using fluorescent microscopy (Fig. 2).

4. Determine the IU of the pseudotype. The IU is defined as a virus titer endpoint determined by limiting dilution.

\subsection{Neutralization Assay of VSV-SARS-St19/GFP Infection Using Antibodies Specific to SARS-CoV}

1. Mix serum samples serially diluted with DMEM-5\%FCS (50 $\mu$ l by volume) with the same volume of DMEM-5\%FCS containing 3000 IU of VSV-SARSSt19/GFP.

2. Incubate for $1 \mathrm{~h}$ at $37^{\circ} \mathrm{C}$ for neutralization.

3. Inoculate the mixture to Vero E6 cells seeded on 96-well culture plates.

4. Follow steps 2 and 3 of Section 3.2.

5. Take photographs of cells expressing GFP under fluorescent microscope.

6. Count the GFP-positive cells on the photographs using the ImageJ software (http://rsb.info.nih.gov/ij/). The results of the neutralization assay using rabbit anti-SARS-CoV antibodies are shown in Fig. 3.

\section{Analyzing Infectivity of VSV Pseudotype as a SEAP Expression}

The infectivity of VSV-SARS-St19/SEAP, for which VSV $\Delta$ G*/SEAP-G is used to produce pseudotype bearing $S$ protein, can be determined as SEAP activities in the culture supernatants.

1. Mix serially diluted VSV-SARS-St19/SEAP with DMEM-5\%FCS and inoculate the mixture into Vero E6 cells seeded on 96-well culture plates (see Note 3).

2. Incubate the cells in a $\mathrm{CO}_{2}$ incubator for $1 \mathrm{~h}$.

3. Remove the inoculum, wash the cells with PBS three times, and then add $100 \mu \mathrm{l}$ of fresh DMEM-5\%FCS (see Note 4). 


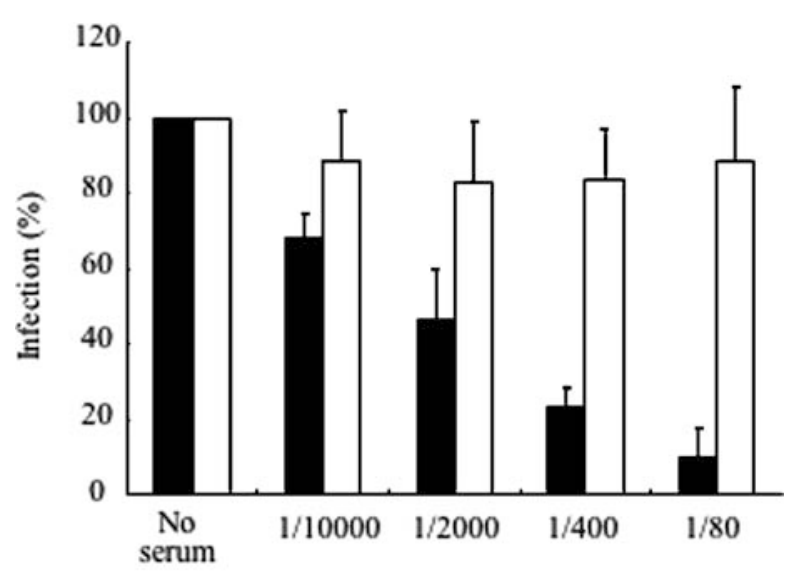

Antibody dilution

Fig. 3. Neutralization of infection of VSV-SARS-St19/GFP. The VSV-SARSSt19/GFP mixed with serially diluted rabbit anti-SARS-CoV was inoculated into Vero E6 cells. Rabbit anti-SARS-CoV-N peptide was used as negative control serum. The number of GFP-positive cells in the absence of serum was set as $100 \%$.

4. Incubate the cells in a $\mathrm{CO}_{2}$ incubator overnight.

5. Determination of SEAP activity is performed by a specific SEAP assay kit. Several kits from different manufacturers are now available, and since manufacturers' protocol details vary, only a general description follows based on the Toyobo kit we have used.

The technique uses 96-well plates. Twenty $\mu$ l of supernatants from cell cultures are added to the wells with an equal volume of endogenous alkaline phosphatase inhibitor. Incubate the mixture at $37^{\circ} \mathrm{C}$ for $30 \mathrm{~min}$. Next, add $160 \mu \mathrm{l}$ of chemiluminescent substrate to the mixture. An incubation of $30 \mathrm{~min}$ at $37^{\circ} \mathrm{C}$ allows the chemiluminescence reaction. Use a luminescence microplate reader to detect the chemiluminescence signal.

\section{Notes}

1. To generate high virus titer of VSV pseudotype, prepare the plasmid encoding a C-terminal truncated version of the $\mathrm{S}$ protein, because it has been shown that truncation of C-terminal 19 amino acids leads to efficient incorporation of $\mathrm{S}$ protein into the VSV particles, and then the VSV pseudotype shows an efficient infection to target cells (11).

2. The VSV $\Delta \mathrm{G}^{*} / \mathrm{GFP}-\mathrm{G}$ is a VSV-G protein-bearing VSV pseudotype in which VSV-G gene is replaced with GFP gene. The VSV $\Delta \mathrm{G}^{*} / \mathrm{SEAP}-\mathrm{G}$ is same as VSV $\Delta G * / G F P-G$ except for having SEAP gene instead of the GFP gene. 
Pseudotypes having VSV-G protein are used as "seed" viruses for generating S protein bearing VSV pseudotypes. Since the VSV $\Delta \mathrm{G}^{*} / \mathrm{GFP}$ or VSV $\Delta \mathrm{G}^{*} / \mathrm{SEAP}$ system was developed by Prof. M. A. Whitt (University of Tennessee Health Science Center, TN), ask him for sharing and using the system when starting experiments.

3. As the SEAP gene is obtained by modification of human placental alkaline phosphatase gene, some cell lines derived from placenta that express alkaline phosphatase similar to SEAP should not be used as target cells. For the analysis of VSV-SARS-St19/SEAP infection, we suggest using Vero E6 cells that show a very low level of alkaline phosphatase activity in the culture supernatant.

4. The medium containing VSV-SARS-St19/SEAP may have strong SEAP activity since it is derived from culture medium of $293 \mathrm{~T}$ cells inoculated VSV $\Delta \mathrm{G}^{*}$ / SEAP-G (see steps 3-6 in Section 3.1). In order to remove carryover SEAP activities derived from VSV $\Delta \mathrm{G}^{*} / \mathrm{SEAP}-\mathrm{G}$ the Vero E6 cells have to be washed with PBS at least three times.

\section{Acknowledgments}

We thank Dr. M. A. Whitt for providing the VSV $\Delta \mathrm{G}^{*} / \mathrm{GFP}-\mathrm{G}$ or VSV $\Delta \mathrm{G}^{*} /$ SEAP-G. This work was supported in part by a grant from the Ministry of Health, Labor, and Welfare of Japan.

\section{References}

1. Li, W, Moore, M. J., Vasilieva, N., et al. (2003) Angiotensin-converting enzyme 2 is a functional receptor for the SARS coronavirus. Nature 426, 450-454.

2. Hofmann, H., Geier, M., Marzi, A., et al. (2004) Susceptibility to SARS coronavirus $S$ protein-driven infection correlates with expression of angiotensin converting enzyme 2 and infection can be blocked by soluble receptor. Biochem. Biophys. Res. Commun. 319, 1216-1221.

3. Nie, Y., Wang, P., Shi, X., et al. (2004) Highly infectious SARS-CoV pseudotyped virus reveals the cell tropism and its correlation with receptor expression. Biochem. Biophys. Res. Commun. 321, 994-1000.

4. Simmons, G., Reeves, J.D., Rennekamp, A.J., Amberg, S.M., Piefer, A.J., Bates, P. (2004) Characterization of severe acute respiratory syndrome-associated coronavirus (SARS-CoV) spike glycoprotein-mediated viral entry. Proc. Natl. Acad. Sci. USA 101, 4240-4245.

5. Matsuura, Y., Tani, H., Suzuki, K., et al. (2001) Characterization of pseudotype VSV possessing HCV envelope proteins. Virology 286, 263-275.

6. Ogino, M., Ebihara, H., Lee, B. H., et al. (2003) Use of vesicular stomatitis virus pseudotypes bearing Hantaan or Seoul virus envelope proteins in a rapid and safe neutralization test. Clin. Diagn. Lab. Immunol. 10, 154-160.

7. Takada, A., Robison, C., Goto, H., et al. (1997) A system for functional analysis of Ebola virus glycoprotein. Proc. Natl. Acad. Sci. USA 94, 14764-14769. 
8. Tatsuo, H., Okuma, K., Tanaka, K., et al. (2000) Virus entry is a major determinant of cell tropism of Edmonston and wild-type strains of measles virus as revealed by vesicular stomatitis virus pseudotypes bearing their envelope proteins. J. Virol. 74, 4139-4145.

9. Moore, M. J., Dorfman, T., Li, W., et al. (2004) Retroviruses pseudotyped with the severe acute respiratory syndrome coronavirus spike protein efficiently infect cells expressing angiotensin-converting enzyme 2. J. Virol. 78, 10628-10635.

10. Nie, Y., Wang, G., Shi, X., et al. (2004) Neutralizing antibodies in patients with severe acute respiratory syndrome-associated coronavirus infection. J. Infect. Dis. 190, 1119-1126.

11. Fukushi, S., Mizutani, T., Saijo, M., et al. (2005) Vesicular stomatitis virus pseudotyped with severe acute respiratory syndrome coronavirus spike protein. J. Gen. Virol. 86, 2269-2274. 